\title{
Unrestricted availability of a plasma paracetamol assay service resulting in an increased number of inappropriate requests
}

\author{
Thomas YK Chan, Julian AJH Critchley, CS Ho, Albert YW Chan
}

\begin{abstract}
Summary
Previously, prior approval from the oncall chemical pathologist was required in our hospital for plasma paracetamol measurements. However, since May 1992, there have been no restrictions on ordering this assay. We have assessed the consequences of this policy change by comparing the number and appropriateness of requests for plasma paracetamol measurements in Chinese patients admitted to our hospital with acute poisoning over two six-month periods (July-December) in 1991 and 1993. Requests were considered appropriate if paracetamol ingestion was suspected or unknown drugs were ingested. The number of patients having plasma paracetamol concentrations assayed increased from 51 in 1991 to 141 in 1993 $(176 \%)$. The corresponding increase in the number of Chinese patients admitted to two of our eight general wards with poisoning was estimated to be $93 \%$. The proportion of 'appropriate' plasma paracetamol measurements dropped from $55 \%$ in 1991 to $21 \%$ in 1993 . Eight patients had plasma paracetamol concentrations above the recommended treatment line; they were all from the group in whom the requests were appropriate. Three of the 135 patients in the group with 'inappropriate' requests were found to have slightly elevated but far from toxic plasma paracetamol concentrations. Unrestricted availability of plasma paracetamol measurements resulted in an increase in the number of inappropriate requests.
\end{abstract}

University of Hong

Kong, Prince of Wales Hospital, Shatin, New

Territories, Hong

Kong

Department of

Clinical

Pharmacology

TYK Chan

JAJH Critchley

Department of

Chemical Pathology

CS Ho

AYW Chan

Accepted 1 June 1995 with acute poisoning for potentially toxic plasma concentrations of paracetamol is therefore not indicated.

Our laboratory at the Prince of Wales Hospital has been providing a 24-hour service for the measurement of plasma paracetamol concentrations since 1984. Results are available within two hours if requested urgently. In May 1992 , it became no longer necessary to obtain prior approval from the on-call chemical pathologists for such requests. The consequences of this policy change on the number of inappropriate requests for plasma paracetamol measurements were determined in the present study.

\section{Subjects and methods}

The Prince of Wales Hospital is the sole genera teaching hospital in the New Territories East of Hong Kong, serving a population of 1.1 million in 1994. During two six-month periods (July-December 1991 and 1993), all patients presenting to our general medical wards with acute poisoning and having had plasma paracetamol measurements performed, were identified from the registry of requests in the Department of Chemical Pathology.

The hospital records of these patients were reviewed. Demographic data and information regarding diagnosis, reason for poisoning, agents involved, treatment given and outcome were noted. The diagnosis of poisoning and identification of agents responsible were based on the history from the patient or witnesses, labelling of the bottles and/or the clinical features.

Based on the findings from our previous study of the clinical value of screening for paracetamol in 294 Chinese patients presenting to our general medical wards between January 1992 and June $1993,{ }^{2}$ we defined requests to be appropriate if paracetamol ingestion was suspected. This included patients who ingested unknown drugs for the treatment of pains, fever, common cold and other upper respiratory tract infections. Since it is reasonable to check for possible paracetamol poisoning in patients who have ingested unknown drugs and unconsious patients, the requests in these patients were also classed as appropriate.

In our 24-hour urgent laboratory, plasma paracetamol concentrations are measured by 
the TDX method (Abbott Laboratories, USA). The detection limit of this method is $0.1 \mathrm{mmol} / 1$.

\section{Results}

During the study periods, the number of Chinese patients having plasma paracetamol concentration measured increased from 51 in 1991 to 141 in 1993 , an increase of $176 \%$. The corresponding increase in the number of Chinese patients admitted to two of our eight general wards with poisoning was estimated to be $93 \%$. However, the proportion of 'appropriate' plasma paracetamol measurements dropped from $55 \%$ in 1991 to $21 \%$ in 1993 (table 1 ).

During the study periods, eight patients had plasma paracetamol levels above the recommented treatment line ${ }^{4}$ (table 2); they were all from the groups in whom the requests were deemed appropriate. Three of the 135 patients in the group with inappropriate requests were found to have slightly elevated but far from toxic plasma paracetamol concentrations which were well below the recommended treatment line.

\section{Discussion}

In this study, we have again shown that routine screening of all patients with acute poisoning for toxic plasma paracetamol concentrations is

Table 1192 Chinese patients with or without a history of paracetamol poisoning admitted to a general teaching hospital over two six-month periods before (1991) and after (1993) the introduction of unrestricted access to the 24-hour plasma paracetamol assay service

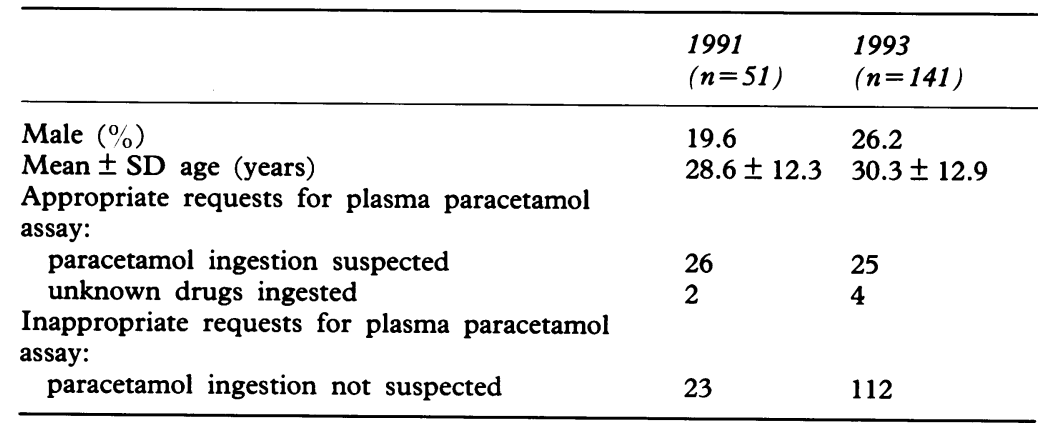

Table 2 Plasma paracetamol measurements in two groups of Chinese patients before (1991) and after (1993) the introduction of unrestricted access to the 24-hour plasma paracetamol assay service

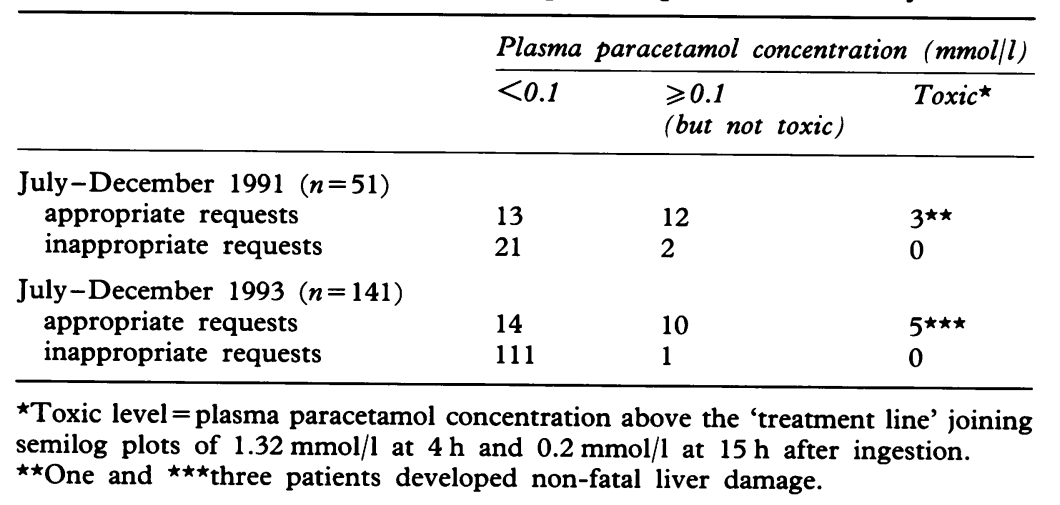

not necessary. Approximately one in seven patients in whom plasma paracetamol measurement was appropriate had potentially hepatotoxic concentrations, and one in 35 patients in whom a plasma paracetamol measurement was not indicated had elevated, but far from toxic concentrations. These figures are comparable to those (one in 11 and one in 36 , respectively) from our previous study. ${ }^{2}$

Prior to May 1992, the requests for plasma paracetamol measurements were considered by our chemical pathologists to be in general quite 'reasonable'. Removing the restrictions on ordering such requests was intended to improve the availability of the test and spare the busy duty chemical pathologist from timeconsuming telephone calls previously considered a mere formality. However, after the test became freely available in May 1992, the number of requests had almost tripled by 1993 compared to 1991. This magnitude of increase could not be accounted for by the increase in the number of patients admitted with acute poisoning. One contributory factor identified in this study was the absolute as well as relative increase in the number of inappropriate requests (see table 1 ).

We cannot be certain why there was an increase in the number of inappropriate requests for plasma paracetamol assays between 1991 and 1993. However, after the removal of restrictions in 1992, this 24-hour assay service became freely available and 'paracetamol screening' became part of the 'routine' investigations for patients with acute poisoning. Some clinicians appeared ignorant of the contents of many prescription drugs as well as the over-the-counter drugs which had been taken by their patients. For example, assay requests were frequently made for patients who had taken adult 'Cortal' (acetylsalicylic acid, caffeine). Confusion also arose because of the difference in content between adult and paediatric preparations of the same product. For example, 'Cortal' for children

Clinical features of paracetamol poisoning

- no specific early symptoms or signs

- nausea and vomiting may occur

- without treatment, severe liver damage occurs in $<10 \%$ of unselected patients, about $1 \%$ suffer acute renal failure, $1-2 \%$ die in hepatic failure

- ethnic difference in susceptibility, eg, renal failure is uncommon in Chinese patients ${ }^{6}$

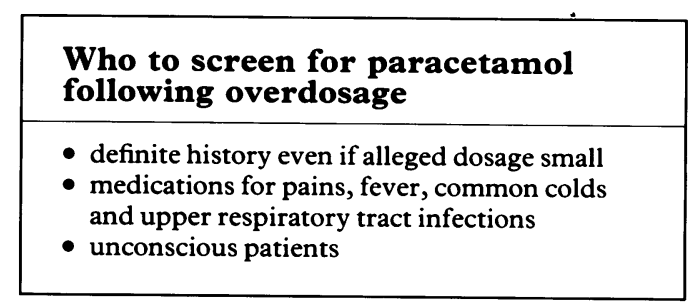




\begin{tabular}{|l|}
\hline Summary/learning points \\
\hline - routine screening of all patients with acute \\
poisoning for toxic plasma paracetamol \\
concentrations is unnecessary \\
unrestricted availability of plasma \\
paracetamol measurements results in an \\
increase in the number of inappropriate \\
requests \\
\hline
\end{tabular}

does contain paracetamol and not the salicylate found in the adult preparation.

We have now proposed guidelines on the use of the plasma paracetamol assay service in our medical unit and we hope to see a reduction in the number of inappropriate requests. The requirement to discuss the case with the duty

1 Chan TYK, Critchley JAJH, Chan MTV, Yu CM. Drug overdosage and other poisoning in Hong Kong - the Prince of Wales Hospital (Shatin) experience. Hum Exp Toxicol 1994, 13: 512-5.

2 Chan TYK, Chan AYW, Ho CS, Critchley JAJH. The clinical value of screening for paracetamol in patients with acute poisoning. Hum Exp Toxicol 1995; 14: 187-9.

3 Ashbourne JF, Olson KR, Khayam-Bashi H. Value of rapid

screening for drug overdose. Ann Emerg Med 1989; 18: 1035-8. chemical pathologists and, perhaps, a clinical toxicologist is now being reconsidered. Such an arrangement also has the advantage of a team approach, particularly in the management of patients with severe poisoning. A comprehensive list of drugs or herbal (patent) medicines containing paracetamol should be readily available to the admitting physicians. Such information is always available from our 24hour Drug and Poisons Information Bureau in Hong Kong. ${ }^{5}$

We have not included the smaller number of non-Chinese subjects (eg, Vietnamese) encountered in this study to avoid the possible confounding factor of the language barrier. Future studies should examine the appropriate as well as inappropriate uses of other drug assays in patients with acute poisoning.

4 Prescott LF, Illingworth RN, Critchley JAJH, et al. Intravenous $\mathrm{N}$-acetylcysteine: the treatment of choice for paracetamol poisoning. BMF 1979; 2: 1097-100.

5 Chan TYK, Critchley JAJH, Chan JCN, et al. The Drug and Poisons Information Bureau at the Chinese University of Hong Kong. 7 Hong Kong Med Assoc 1993; 45: 202-5.

6 Chan TYK, Critchley JAJH, Chan AYW. Renal failure is uncommon in Chinese patients with paracetamol (acetaminophen) poisoning. Vet Hum Toxicol 1995; 37: 154-6.

\section{Grants for travel and research 1996}

The Fellowship of Postgraduate Medicine is offering grants for travel, or towards the cost of equipment, for young graduates entering careers in clinical research. These are intended as 'top-up' grants for existing research, or for specific purposes of travel in connection with the research, and are of the order of $£ 1000$.

The application should give a concise summary of the research project, the sum requested, details of the equipment or of the travel project, and include a curriculum vitae of the applicant.

Successful applicants will be expected to submit reports of their research for publication in the Postgraduate Medical fournal.

Applications should be sent to:

Mrs JM Coops

Executive Secretary

Fellowship of Postgraduate Medicine

12 Chandos Street

London W1M 9DE, UK 